\title{
Insect swarms can be bound together by repulsive forces ${ }^{\star}$
}

\author{
A.M. Reynolds ${ }^{\mathrm{a}}$ \\ Rothamsted Research, Harpenden, Hertfordshire, AL5 2JQ, UK
}

Received 9 January 2020 and Received in final form 1 June 2020

Published online: 19 June 2020

(C) The Author(s) 2020. This article is published with open access at Springerlink.com

\begin{abstract}
The cohesion of insect swarms has been attributed to the fact that the resultant internal interactions of the swarming insects produce, on the average, a centrally attractive force that acts on each individual. Here it is shown how insect swarms can also be bound together by centrally forces that on the average are repulsive (outwardly directed from the swarm centres). This is predicted to arise when velocity statistics are heterogeneous (position-dependent). Evidence for repulsive forces is found in laboratory swarms of Chironomus riparius midges. In homogeneous swarms, the net inward acceleration balances the tendency of diffusion (stochastic noise) to transport individuals away from the centre of the swarm. In heterogenous swarms, turbophoresis - the tendency for individuals to migrate in the direction of decreasing kinetic energy - is operating. The new finding adds to the growing realization that insect swarms are analogous to self-gravitating systems. By acting in opposition to central attraction (gravity), the effects of heterogeneous velocities (energies) are analogous to the effects of dark energy. The emergence of resultant forces from collective behaviours would not be possible if individual flight patterns were themselves unstable. It is shown how individuals reduce the potential for the loose of flight control by minimizing the influence of jerks to which they are subjected.
\end{abstract}

\section{Introduction}

In contrast with bird flocks, fish schools and migratory herds, sparse swarms of flying insects do not possess global order but are, nonetheless, a form of collective animal behaviour $[1,2]$. The collective behaviour is evident in their emergent macroscopic mechanical properties. Laboratory swarms of Chironomus riparius midges, for example, have macroscopic mechanical properties similar to solids, including a finite Young's modulus and yield strength [3]. The collective behaviour of these swarms is also evident in their response to dynamic illumination perturbations. The swarm-level response can be described by making an analogy with classical thermodynamics, with the state of the swarm moving along an isotherm in a thermodynamic phase plane [4]. Applied oscillatory visual stimuli induce a viscoelastic response as the perturbations are strongly dampened, both viscously and inertially [5]. A distinctively different indicator of collective behavior lies in the composition of unperturbed swarms. Unperturbed laboratory swarms of Chironomus riparius midges consist of a core "condensed" phase surrounded by a dilute "vapour" phase [6]. Although these two phases have distinct macroscopic properties, individuals move freely between them,

\footnotetext{
* Supplementary material in the form of a .pdf file available from the Journal web page at

https://doi.org/10.1140/epje/i2020-11963-x

a e-mail: andy.reynolds@rothamsted.ac.uk
}

suggesting that they are collective, emergent states. The collective behaviours of laboratory swarms of Chironomus riparius midges are predicted by stochastic trajectory simulation models [5,7-11]. This and other modelling [12-14] have also uncovered striking similarities between insect swarms and self-gravitating systems such as globular clusters, as foreseen by Okubo [1]. Okubo [1] noted that if the internal forces between individuals were like Newtonian gravitational attraction, then the resultant attraction on an individual within a uniform spherical swarm would be directly proportional to the distance from the swarm centre, as observed $[1,2]$ and as predicted by the stochastic models.

To date, stochastic trajectory simulation models have been formulated for homogeneous swarms with positionindependent velocity statistics. Here models are formulated for swarms with heterogeneous velocity statistics. The effects of heterogeneous velocity statistics are shown to be analogous to "dark energy" causing individuals, on the average, to accelerate outwardly from the swarm centre. The outward accelerations need a supply of energy which for "active particles" like insects can be got from converting (unseen) internal energy into kinetic energy. Model predictions are supported by the results of numerical simulations and by analysis of pre-existing data for laboratory swarms of Chironomus riparius midges [15].

Swarm stability is contingent on the stability of individual trajectories. Modelling and data analysis reveal 
that individuals minimize the influence of potentially destabilizing jerks (changes in acceleration) to which they are subjected. I show that jerks along with an analogue of the Reynolds number appear in higher-order (generalized) stochastic models.

\section{Model formulation and predictions}

Here following Okubo [1] I assume that the positions, $x$, and velocities, $u$, of individual insects within a swarm can be described by the stochastic differential equations

$$
\begin{aligned}
\mathrm{d} x & =u \mathrm{~d} t, \\
\mathrm{~d} u & =a(u, x, t) \mathrm{d} t+b \mathrm{~d} W(t),
\end{aligned}
$$

where $\mathrm{d} W(t)$ is an incremental Wiener process with correlation property $\overline{\mathrm{d} W(t) \mathrm{d} W(t+\tau)}=\delta(\tau) \mathrm{d} t$. Such 1dimensional, individual-based models are effectively firstorder autoregressive stochastic processes in which positions and velocities are modelled as a joint Markovian process. At second order, positions, velocities and accelerations are modelled collectively as a Markovian process. Physically, the hierarchy of stochastic models corresponds to the inclusion of a velocity autocorrelation timescale, $T$, at first order, and to the addition of an acceleration autocorrelation timescale, $t_{A}$, at second order and so on [16]. Continuum models of the kind pioneered by Topaz and Bertozzi [17] and utilized, for example, by Topaz et al. [18] are not appropriate because the Knudsen number $\mathrm{Kn} \sim \mathrm{O}(1)$ [19]. In the laboratory, Chironomus riparius midges appear somewhat paradoxically to be tightly bound to the swarm while at the same time weakly coupled inside it [19].

Here the deterministic term, $a(u, x, t)$, is determined by the requirement that the statistical properties of the simulated trajectories be consistent with the observations of Kelley and Ouellette [2] who reported on the position and velocity statistics of individual Chironomus riparius midges within laboratory swarms. Mathematically these consistency conditions require that the joint distribution of velocity and position $p(u, x, t)$ be a solution of the Fokker-Planck equation

$$
\frac{\partial p}{\partial t}+u \frac{\partial p}{\partial x}=-\frac{\partial}{\partial u}(a p)+\frac{b^{2}}{2} \frac{\partial^{2} p}{\partial u^{2}} .
$$

Here, in broad agreement with the observations [2], I assume that positions and velocities are statistically stationary and Gaussian distributed,

$$
p(u, x)=\frac{1}{2 \pi \sigma_{x} \sigma_{u}} \exp \left(-\frac{x^{2}}{2 \sigma_{x}^{2}}\right) \exp \left(-\frac{u^{2}}{2 \sigma_{u}^{2}}\right),
$$

where $\sigma_{x}$ is the root-mean-square position (i.e., the root-mean-square swarm size), and $\sigma_{u}$ is the positiondependent root-mean-square speed. Equation (2) implies

$$
a p=\frac{b^{2}}{2} \frac{\partial p}{\partial u}+\phi(x, u, t)
$$

where for statistically stationary swarms having, $\frac{\partial p}{\partial t}=0$, the quantity $\phi$ is determined by

$$
\frac{\partial \phi}{\partial u}=-u \frac{\partial p}{\partial x}, \quad \text { i.e., } \quad \phi=-\frac{\partial}{\partial x} \int_{-\infty}^{u} u p \mathrm{~d} u .
$$

It follows from eqs. (2), (3) and (4) that

$$
\mathrm{d} u=-\frac{u}{T} \mathrm{~d} t-\frac{\sigma_{u}^{2}}{\sigma_{x}^{2}} x \mathrm{~d} t+\frac{1}{2}\left(1+\frac{u^{2}}{\sigma_{u}^{2}}\right) \frac{\mathrm{d} \sigma_{u}^{2}}{\mathrm{~d} x} \mathrm{~d} t+\sqrt{\frac{2 \sigma_{u}^{2}}{T}} \mathrm{~d} W
$$

when, without loss of generality and on dimensional grounds, $b=\sqrt{\frac{2 \sigma_{u}^{2}}{T}}$ where $T$ is a model timescale. Details of the derivation of such models can be found in Thomson [20]. The first term in eq. (5) is a "memory term" which causes velocity fluctuations to relax to their mean value. The second and third terms are a conditional mean acceleration (restorative force). The fourth term is the stochastic driving noise. This accounts for fluctuations in the restorative force which arise because of the limited number of individuals in the swarm and because of nonuniformity in their spatial distribution [1]. Utilizing the continuous Fokker Planck equation, eq. (2), to determine the functional form of the discrete models, eq. (1), in the above way contrasts with the continuum formulation of fully-fledged discrete models of dense swarms [21].

When velocities are homogeneous (i.e., when $\frac{\mathrm{d} \sigma_{u}^{2}}{\mathrm{~d} x}=0$ ), the model, eq. (5), reduces to Okubo's model [1]. In this case, mean accelerations are directed towards the swarm centre and increase linearly with distance from the swarm centre, in accordance with observations made at the cores of laboratory swarms $[1,2]$. This is consistent with insect swarms behaving as self-gravitating systems [1].

More generally, the velocity-averaged mean acceleration

$$
\langle A \mid x\rangle=-\frac{\sigma_{u}^{2}}{\sigma_{x}^{2}} x+\frac{\mathrm{d} \sigma_{u}^{2}}{\mathrm{~d} x} .
$$

Concave mean-square-velocity profiles therefore counteract the linear term, $-\frac{\sigma_{u}^{2}}{\sigma_{x}^{2}} x$, (more generally $\sigma_{u}^{2} \frac{\mathrm{d} \rho}{\mathrm{d} x}$ where $\rho$ is the aerial density profile) and if strong enough can overwhelm it completely so that mean accelerations are everywhere direct away rather than towards the swarm centre. Such swarms are therefore effectively bound together by repulsive forces.

\section{Comparisons with simulation and experimen- tal data}

Individual trajectories were simulated by numerically integrating the stochastic model, eqs. (1) and (5). Statistically stationary predictions for mean accelerations, velocity variances and spatial distributions were obtained from 100000 simulated trajectories. The results of these numerical simulations confirm that swarms remain localized and coherent even though individuals are, on the average, accelerating outwardly away from the swarm centre. This is 
(a)

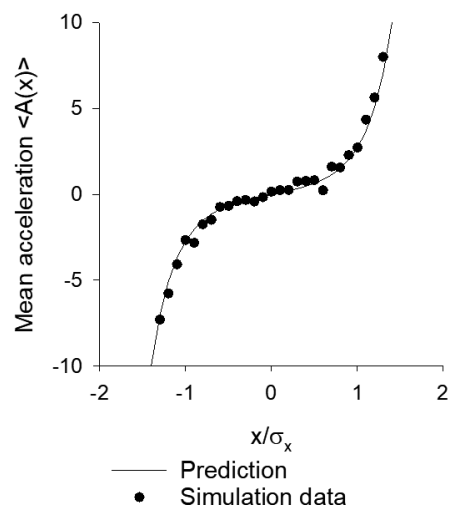

(b)

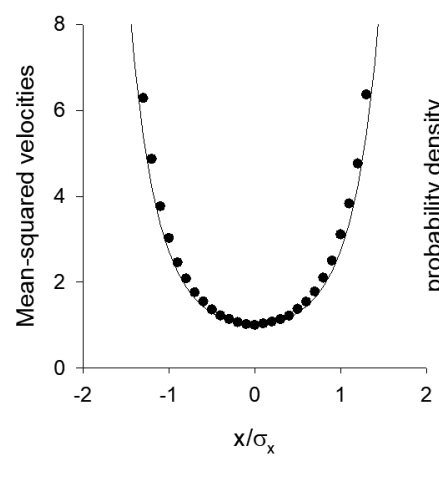

(c)

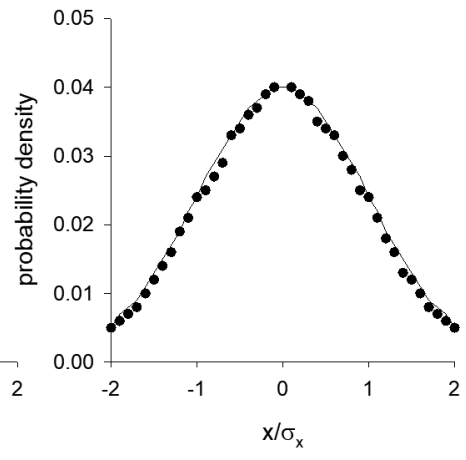

Fig. 1. Insect swarms are predicted to remain localized and coherent even when every individual is, on the average, accelerating outwardly from the swarm centre. Individual trajectories were simulated using the stochastic model, eqs. (1) and (5) with $\sigma_{x}^{2}=1, \sigma_{u}^{2}=\sigma_{0}^{2} \mathrm{e}^{x^{2} / \sigma_{x}^{2}}$ for $|x|<2 \sigma_{x}$ otherwise $\sigma_{u}^{2}=\sigma_{0}^{2} e^{4}, \sigma_{0}=1$ and $T=1 \mathrm{a} . \mathrm{u} .100000$ individuals were simulated for a time $t=5$ a.u. whereupon their positions, velocities and accelerations were recorded $(\bullet)$. Initial positions were Gaussian distributed with mean zero and variance $\sigma_{x}^{2}$. Initial velocities were Gaussian distributed with mean zero and variance $\sigma_{u}^{2}(x)$.

illustrated in fig. 1 for the case when $\sigma_{u}^{2}=\sigma_{0}^{2} \mathrm{e}^{x^{2} / \sigma_{x}^{2}}$ for $|x|<2 \sigma_{x}$ otherwise $\sigma_{u}^{2}=\sigma_{0}^{2} e^{4}$ so that $\langle A \mid x\rangle=\frac{\sigma_{0}^{2}}{\sigma_{x}^{2}} x$ for $|x|<2 \sigma_{x}$ otherwise $\langle A \mid x\rangle=0$. Cases where individuals are everywhere, on the average, repulsive are non-physical because such swarms possess infinite kinetic energy. Further support for the model predictions comes from an analysis of the pre-existing data for 3-dimensional swarms of Chironomus riparius midges [15]. Mean-square velocities within the cores of these swarms are position independent and, as predicted, mean accelerations increase linearly with distance from the swarm centers (fig. 2). In the outskirts of the swarms the concave shape of meansquare velocity profiles becomes apparent as does the expected associated weakening of the central attraction (due to contributions to the net resultant forces from repulsive forces).

\section{Jerks and Reynolds numbers}

Male midges swarm to provide a mating target for females, making stationarity desirable. Ni and Ouellette [3] were the first to show that this biological function is reflected in an emergent physical macroscopic property of the swarm; namely its tensile strength. van der Vaart et al. [5] subsequently showed that midge swarms also strongly dampen perturbations, both viscously and inertially. These findings suggest that midge swarms use their collective behaviour to stabilize themselves against environmental perturbations. Perturbations are inevitable in natural swarms that must contend with gusts of wind and with other environmental disturbances. Collective behavours can, however, only be stablizing if individual's trajectories are themselves stable. To avoid losing control of their body motion, it is not only necessary to limit the maximum acceleration, i.e., the force, an individual can be exposed to, but also the maximum jerk strength (rate of change of acceleration, $\left.\frac{\mathrm{d}^{3} x}{\mathrm{~d} t^{3}}\right)$, since individuals need time to adjust to stress changes. Here I show that midges minimize the impact of jerks.

Jerks arise in second-order autoregressive models for the joint evolution of an individual's position, $x$, velocity, $v$, and acceleration, $A$ :

$$
\begin{aligned}
\mathrm{d} A & =a(A, u, x, t) \mathrm{d} t+b \mathrm{~d} W(t), \\
\mathrm{d} u & =A \mathrm{~d} t, \\
\mathrm{~d} x & =u \mathrm{~d} t .
\end{aligned}
$$

The formulation of such models mirrors closely that of first-order models, eq. (1). The position, velocity and accelerations of the simulated trajectories will be consistent with the observed form of the joint distribution of acceleration, velocity and position, $P(A, u, x, t)$, when $P(A, u, x, t)$ is a solution of the Fokker-Planck equation

$$
\frac{\partial P}{\partial t}+u \frac{\partial P}{\partial x}+A \frac{\partial P}{\partial u}=-\frac{\partial}{\partial A}(a P)+\frac{b^{2}}{2} \frac{\partial^{2} P}{\partial A^{2}} .
$$

Equation (8) implies that

$$
a=\frac{b^{2}}{2} \frac{\partial \ln P}{\partial A}+\frac{\phi}{P},
$$

where for statistically stationary swarms having, $\frac{\partial P}{\partial t}=0$,

$$
\frac{\partial \phi}{\partial A}=-u \frac{\partial P}{\partial x}-A \frac{\partial P}{\partial u}
$$

The first term on the right-hand side of eq. (9) is a memory term which causes accelerations to relax to their mean value, $\langle A\rangle$. The second term on the right-hand side $\frac{\phi}{P}$ of eq. (9) is the mean jerk strength $\langle J\rangle$. It follows from eq. (10) that when accelerations, velocities are homogeneous (position independent) and Gaussian distributed, the mean jerk strength

$$
\langle J\rangle=u\left(\frac{\partial\langle A\rangle}{\partial x}-\frac{\sigma_{A}^{2}}{\sigma_{u}^{2}}\right) .
$$


(a)

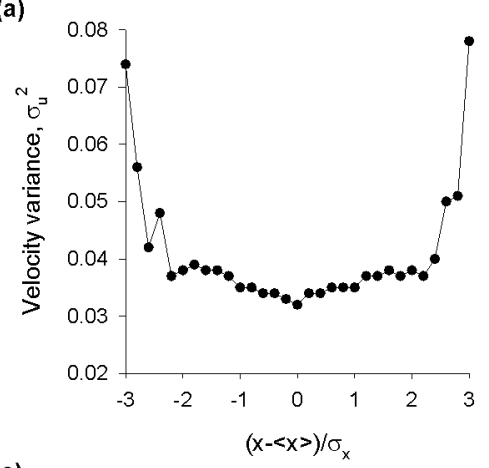

(c)

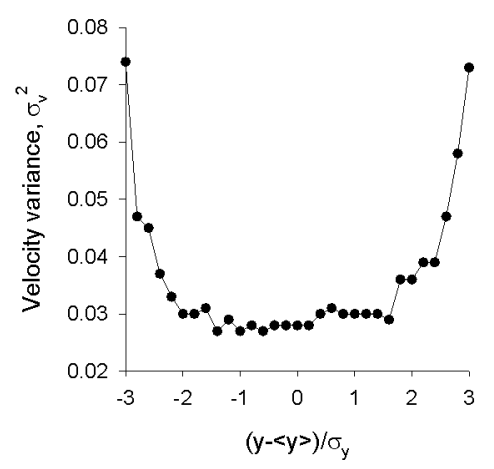

(b)

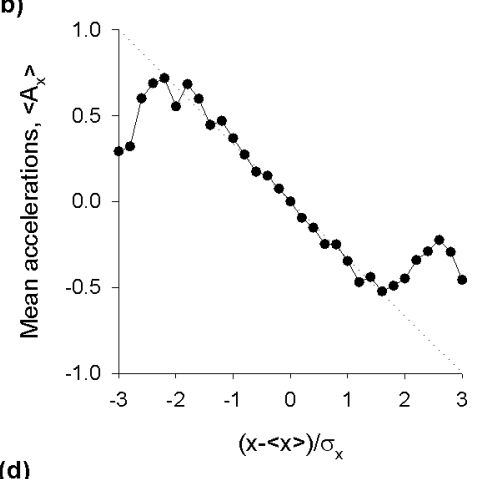

(d)

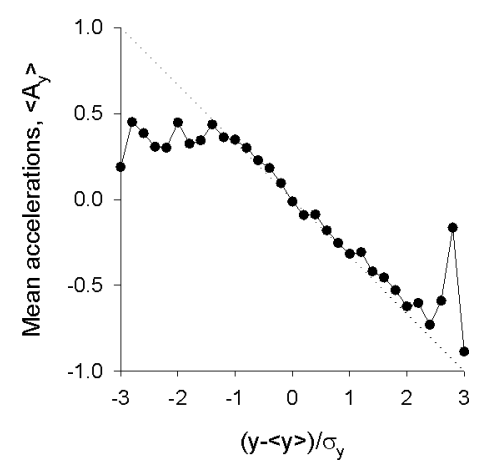

Fig. 2. Hallmarks of model predictions in laboratory insect swarms. Concave velocity-variance profiles co-exist with suppressed inward accelerations (indicative of contributions from outward accelerations). Data are taken from Sinhuber et al. [15] for horizontal movements. Velocity variances and mean accelerations are ensemble averages over all 17 dusk time swarms in the dataset. To reduce the effects of meandering centres and fluctuations in swarm size, position-dependent velocity variances and mean accelerations were calculated for $10 \mathrm{~s}$ long runs of data. These quantities were then ensemble-averaged. Data for vertical movements is not shown because laboratory swarms are distorted in that direction by the presence of the ground, and because individuals tend to join the swarm by flying above it [1]. Also shown is the linear dependence of mean accelerations with distance from the swarm centre that is expected for homogeneous swarms (dashed lines).

An equation for the mean acceleration -identical to eq. (4) - is obtained from eq. (10) after integrating over all accelerations. For swarms with Gaussian density profiles, $\langle A\rangle=-\frac{\sigma_{u}^{2}}{\sigma_{x}^{2}} x$. In accordance with eq. (11), the mean jerk strength is observed to increase linearly with velocity when velocities lie within the Gaussian cores of the velocity distributions (fig. 3(a)). Discrepancies between the predicted and observed mean jerk strengths only become significant at higher velocities, $|u|>2 \sigma_{u}$, which lie within the exponential tails of the velocity distribution [2] and so beyond the scope of the model. Moreover, as predicted, the average observed jerk strength does not vary significantly with position in the swarm (fig. 3(b)). Model predictions for velocity-averaged jerk strengths $|\langle J(u>0)\rangle|$ are also in good agreement with data for a variety of laboratory swarms with mean sizes between 19 and 94 individuals (fig. 3(c)). The simple, 1-dimensional model is seen to consistency overpredict $|\langle J(u>0)\rangle|$ by a factor of about $3 / 2$.

The above analysis is readily extended from 1 and 3 dimensions and to thereby account for velocity covariances. In this case

$$
\left\langle J_{i}\right\rangle=-u_{j}\left(\frac{\left\langle u_{i} u_{j}\right\rangle}{\sigma_{x}^{2}}+\sigma_{A}^{2} \tau_{i j}\right)
$$

where the subscripts denote Cartesian coordinates and where $\tau$ is the inverse of the velocity covariance matrix $\left\langle u_{i} u_{j}\right\rangle$. The mean jerks are therefore aligned with the direction of travel, i.e., with the body axis, when the velocity covariances vanish. This has resonance with Reynolds et al. [22] who suggested that migratory insects use turbulence-induced jerks as an indication of mean wind direction when flying at altitude. The average magnitude of the jerks is the smallest along the mean wind line (or to the right of the mean wind line in Ekman spiral atmosphere in the Northern Hemisphere). Vanishing velocity covariances are not inevitable. The analysis of the datasets of Sinhuber et al. [15] of laboratory swarms of midges, for example, reveals that velocity covariances are non-zero but 10 to 20 times smaller than the velocity variances. And, as predicted, mean jerks are found to be effectively aligned with velocity.

An analogue of the Reynolds number, which is determined by the ratio of $T$ and $t_{A}, \operatorname{Re}^{*}=\left(T / t_{A}\right)^{2}$, appears as a parameter at second order [16]. This provide a new way to characterise swarm behaviours. Indeed, modelling predicts a transition from under- and over-damped movements with increasing Reynolds number (fig. S1 in the Electronic Supplementary Material). 
a)

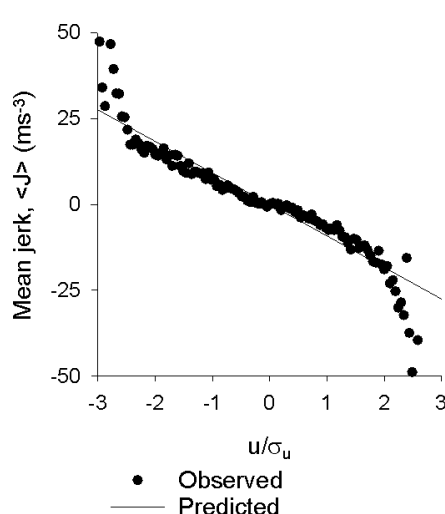

b)

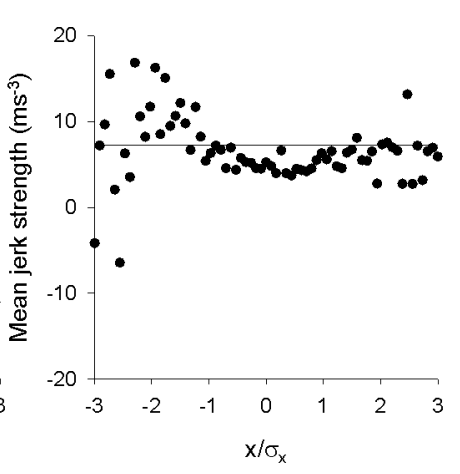

c)

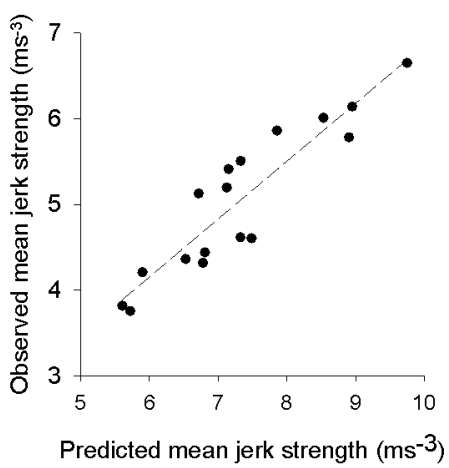

Fig. 3. Comparisons of predicted and observed mean jerk strengths. An example comparison of the predicted and observed dependence of the mean jerk strength on (a) velocity and (b) position. Experimental data are taken from the smallest swarm (Ob17) in the data set of Sinhuber et al. [15]. In accordance with model expectations, eq. (11), the mean jerk strength increases linearly with velocity according to $J=-u\left(\frac{\sigma_{u}^{2}}{\sigma_{x}^{2}}+\frac{\sigma_{A}^{2}}{\sigma_{u}^{2}}\right)$ and the mean jerk strength $|\langle J(u>0)\rangle|=\sqrt{\frac{2}{\pi}} \sigma_{u}\left(\frac{\sigma_{u}^{2}}{\sigma_{x}^{2}}+\frac{\sigma_{A}^{2}}{\sigma_{u}^{2}}\right)$ is independent of position. (c) Comparison of predicted and observed velocity-averaged jerk strengths $|\langle J(u>0)\rangle|$. Experimental data are taken from all 17 dusk-time swarms in the data set of Sinhuber et al. [15]. The dashed-line is a least squares regression $(R=0.93)$. Directly comparable results are obtained for the $y$ - and $z$ - (vertical) directions.
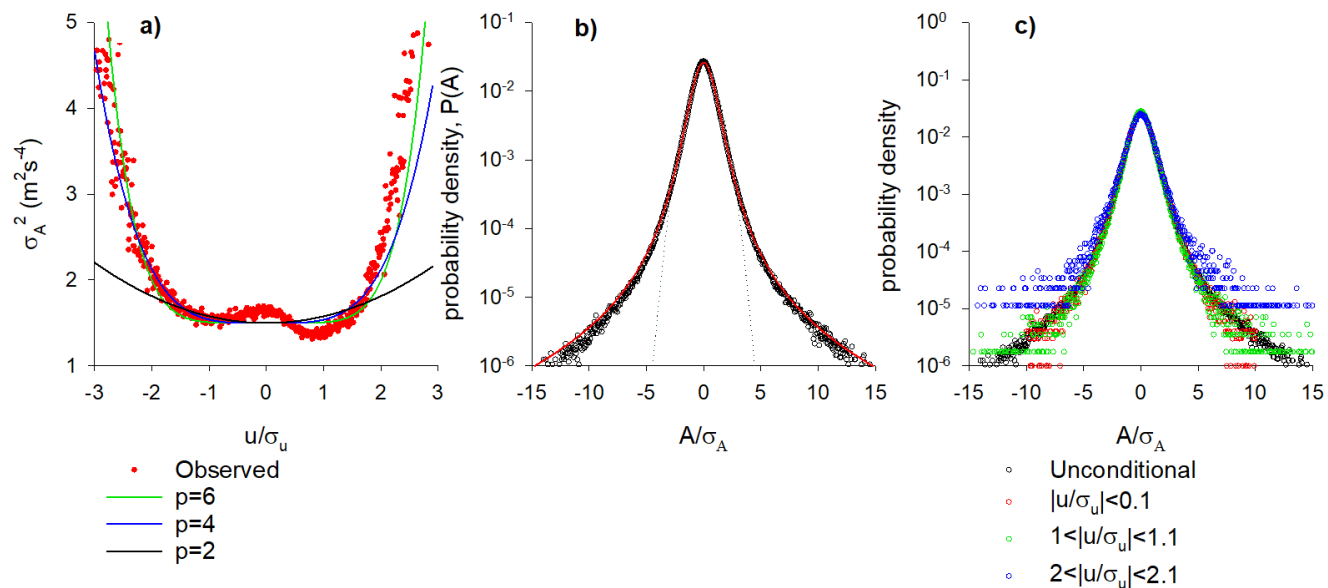

Fig. 4. (a) Acceleration variances depend on velocity. The lines are fits to $\sigma_{A}^{2}=a_{0}+a_{p} u^{p}$ with $p=2,4$ and 6 . Experimental data are taken from all 17 dusk-time swarms in the data set of Sinhuber et al. [15]. (b) Comparison of observed and predicted unconditional probability density functions (PDFs) of acceleration. Experimental data are taken from all 17 dusk-time swarms in the data set of Sinhuber et al. [15] (black line) (one horizontal component of acceleration). In accordance with model expectations the PDF has a heavy tailed compared with the Gaussian (dashed line). The data are very well represented by a stretched exponential $P(A)=N \exp \left(-\frac{A^{2}}{\left(\alpha+\beta|A|^{2}\right)^{6 / 7}}\right)$ where $N$ is a normalization constant and where the constants $\alpha$ and $\beta$ were determined by maximizing the associated log likelihood function (red line). (c) Unconditional and conditional PDFs of acceleration have similar shapes. The observed collapse of the conditional PDFs suggests that the stretched exponential tails and the postulated power law of the conditional acceleration variances may be related.

\section{More elaborate models}

In principle the modelling could be extended to account for distributions of acceleration having heavy tails [2]. It should, however, be noted that the naive approach of simply specifying non-Gaussian accelerations produces nonphysical effects in stochastic modelling of tracer-particle trajectories in turbulence [23]. More realistic stochastic models of tracer-particle trajectories in turbulence are formulated in terms of conditional distributions of accelerations under the assumption that these are Gaus- sian [24-26]. Tracer-particle accelerations have a conditional dependency on both the rate of dissipation of turbulent kinetic energy and velocity [16]. Likewise, the mean accelerations of swarming insects are velocity dependent [7], as are the acceleration variances, $\sigma_{A}^{2}(u, x)$ (fig. 4(a)). The latter implies that large fluctuations in acceleration tend to be associated with large velocities. The correlation between the square of the fluctuations in accelerations and square of velocity is small but not negligible (the correlation coefficient, $\rho_{A^{2} u^{2}}=$ 

$\left.\frac{\left\langle A^{2} u^{2}\right\rangle-\left\langle A^{2}\right\rangle\left\langle u^{2}\right\rangle}{\left(\left\langle A^{4}\right\rangle-\left\langle A^{2}\right\rangle^{2}\right)^{1 / 2}\left(\left\langle u^{4}\right\rangle-\left\langle u^{2}\right\rangle^{2}\right)^{1 / 2}} \sim 0.1\right)$. Fluctuations in acceleration are, therefore, just as likely to reinforce the velocity as they are to oppose it. The data for $\sigma_{A}^{2}(u, x)$ are seen to be equally well represented by $\sigma_{A}^{2}=a_{0}+a_{4} u^{4}$ and by $\sigma_{A}^{2}=a_{0}+a_{6} u^{6}$ (fig. 4(a)). The velocity dependency of the acceleration variances may be attributed to insect trajectories occasionally rotating [10]. An insect will complete a half a rotation (of radius $r$ ) and change its velocity by an amount $\Delta u=2 u$ in a time $\tau=\pi r /|u|$. Consequently, acceleration variances $\left\langle\left(\frac{\Delta u}{\tau}\right)^{2}\right\rangle \sim u^{4}$ when $u \sim r^{0}$ and $\sim u^{6}$ when $u \sim r^{-1}$. If $u \sim r^{-1}$ then an insect will not rotate over itself: it will maintain the same orientation while circulating behaving like a passive particle in an irrotational produced by a vortex tube. It is interesting to note that the acceleration variances of passive tracer-particles in turbulence and those of simulated passive tracer-particles of direct numerical simulations also have a $u^{6}$ dependence which has been attributed to rotations (around vortex filaments) $[27,28]$. As with the case of turbulence [28], the velocity-dependent accelerations variances may account for distributions of acceleration having heavy tails [1] (fig. 4(b)) and for the collapse of the conditional distributions $P(A \mid u)$ (fig. $4(\mathrm{c})$ ). Following Sawford et al. [28], a heuristic understanding of this relationship can be attained by assuming that conditional distributions of acceleration are Gaussian. For large accelerations, the unconditional distribution of accelerations, $P(A)=\int_{-\infty}^{\infty} P(A \mid u) p(u) \mathrm{d} u$ can be evaluated using the saddle point approximation. If, as observed [1], velocity distributions have long exponential tails, then the saddle point approximation gives $P(A) \sim \exp \left(-\alpha A^{\frac{2}{1+p}}\right)$ where $\alpha$ is a constant. Laboratory results for the distribution acceleration are well represented by $P=N \exp \left(-\frac{A^{2}}{\left(\alpha+\beta|A|^{2}\right)^{6 / 7}}\right)$ where $N$ is a normalization constant and where $\alpha$ and $\beta$ are constants (fig. 4(c)). This ansatz has a Gaussian core and a stretched exponential tail. The model exponent for the tail, $2 /(1+p)$, matches the empirical value, $2 / 7$, when $p=6$. It may be difficult to improve on this model. Note also that these intrinsic fluid-like properties of swarming midges in still air may, like other properties of swarming midges, be modified if the air itself is set in motion or if the swarm is perturbed in other ways by external perturbations $[4,5]$; mirroring expectations for swarming in viscous fluids [29].

\section{Discussion}

Here I reported on the first theoretical analysis of heterogeneous insect swarms with position-dependent velocity statistics. The analysis demonstrated the utility of a model formulation which has proved to be highly effective when applied to homogeneous swarms $[5,7-11]$. It revealed how heterogeneous velocity statistics $\frac{\mathrm{d} \sigma_{u}^{2}}{\mathrm{~d} x}$ contribute to mean accelerations, $\langle A \mid x\rangle$, countering or even overwhelming centrally attractive accelerations, $-\frac{\sigma_{u}^{2}}{\sigma_{x}^{2}} x$ (eq. (5)). The former was evident in the results of numerical simulations (fig. 1) which confirmed that individuals can be bounded to the swarm centre by a resultant force that, on the average, is repulsive; a seemingly paradoxical situation. Evidence for repulsive forces was uncovered in an analysis of pre-existing experimental data (fig. 2). The mechanism is clear. In homogeneous swarms, the net inward acceleration balances the tendency of diffusion (stochastic noise) to transport individuals away from the centre of the swarm. In heterogenous swarms, turbophoresis is operating. If, as is observed (fig. 2), $\sigma_{u}^{2}(x)$ is concave, then in statisticallystable swarms this tendency of individuals to move inwards towards to centres of swarms must be countered by net outward accelerations. Concave velocity-variance profiles together with the assumption that velocities are locally Gaussian also accounts for the observed presence of velocity distributions with long-exponential tails [10]. This juxtaposition also accounts for the observed occurrence of speed-dependent forces, eq. (5) [7]. Concave velocityvariance profiles are predicted by the mechanistic models which attribute swarm cohesion to the sporadic and temporary formulation of bound pairs of individuals flying in synchrony [10], as observed by Puckett et al. [30]. They may be also attributed to the influence of the groundbased visual features known as swarm markers [19] or result from interactions between swarming insects and faster insects outside of the swarms [19]: interactions that may also account for the presence of stabilizing inwards effective pressure on the surface of the swarms [12].

Okubo [1] speculated that insect swarms are analogous to self-gravitating systems and therefore individuals are attracted to the centre of the swarm by an effective net force that increases linearly with distance from the swarm centre. There is now strong experimental support for such a net linear restoring force operating within the cores of laboratory swarms [2]. More recent studies have uncovered more striking analogies with self-gravitating systems: including the occurrence of polytropic distributions (which constitute the simplest, physically plausible models for self-gravitating stellar systems), together with biological correlates of Jean's instabilities, black hole entropies, Mach's Principle, surface pressures, and dark matter (see refs. [10,12-14,31] and Electronic Supplementary Material). By providing a revision to Okubo [1] I have uncovered another biological correlate of self-gravitating systems: namely dark energy. In analogy with dark energy, heterogenous velocity statistics were shown to act in opposition to the net inward force identified by Okubo [1]. This opposition becomes significant in the outskirts of swarms. The enrichening of the analogy with self-gravitating systems compliments ongoing attempts to establish a "thermodynamic" understanding of swarming [4,32]. A complete understanding of the collective behaviour of insect swarms may ultimately be found in both their emergent macroscopic mechanical and thermodynamic properties, and in their similitude with self-gravitating systems. The emergence of these properties is contingent on individuals not losing control of their trajectories. It was shown how individuals in the swarms reduce the potential for the loose of flight control by minimizing the potentially destabilizing influences of jerks; mirroring expectations for migratory insects [22]. 
Finally, it was shown that an analogue of the Reynolds number appears as a parameter in second-order stochastic models, opening a new unexplored avenue for characterizing collective behaviours [33].

The work at Rothamsted forms part of the Smart Crop Protection (SCP) strategic programme (BBS/OS/CP/000001) funded through the Biotechnology and Biological Sciences Research Council's Industrial Strategy Challenge Fund.

Publisher's Note The EPJ Publishers remain neutral with regard to jurisdictional claims in published maps and institutional affiliations.

Open Access This is an open access article distributed under the terms of the Creative Commons Attribution License (http://creativecommons.org/licenses/by/4.0), which permits unrestricted use, distribution, and reproduction in any medium, provided the original work is properly cited.

\section{References}

1. A. Okubo, Adv. Biophys. 22, 1 (1986).

2. D.H. Kelley, N.T. Ouellette, Sci. Rep. 3, 1073 (2013).

3. R. Ni, N.T. Ouellette, Phys. Biol. 13, 045002 (2016).

4. M. Sinhuber, K. van der Vaart, N.T. Ouellette, J. R. Soc. Interface 16, 20180739 (2019).

5. K. van der Vaart, M. Sinhuber, A.M. Reynolds, N.T. Ouellette, Sci. Adv. 5, eaaw9305 (2019).

6. M. Sinhuber, N.T. Ouellette, Phys. Rev. Lett. 119, 178003 (2017).

7. A.M. Reynolds, M. Sinhuber, N.T. Ouellette, Eur. Phys. J. E 40, 46 (2017).

8. A.M. Reynolds, J. R. Soc. Interface 15, 20170806 (2018).

9. A.M. Reynolds, Phys. Biol. 16, 046002 (2019).

10. A.M. Reynolds, J. R. Soc. Interface 16, 20190404 (2019).

11. K. van der Vaart, M. Sinhuber, A.M. Reynolds, N.T. Ouellette, J. R. Soc. Interface 17, 20200018 (2020).
12. D. Gorbonos, R. Ianconescu, J.G. Puckett, R. Ni, N.T. Ouellette, N.S. Gov, New J. Phys. 18, 073042 (2016).

13. D. Gorbonos, K. van der Vaart, M. Sinhuber, J.G. Puckett, N.T. Ouellette, A.M. Reynolds, N.S. Gov, Phys. Rev. Res. 2, 013271 (2020)

14. A.M. Reynolds, Europhys. Lett. 124, 38001 (2018).

15. M. Sinhuber, K. van der Vaart, R. Ni, J.G. Puckett, D.H. Kelley, N.T. Ouellette, Sci. Data 6, 190036 (2019).

16. B.L. Sawford, Phys. Fluids A 3, 1577 (1991).

17. C.M. Topaz, A.L. Bertozzi, SIAM J. Appl. Math. 65, 152 (2004).

18. C.M. Topaz, M.R. D’Orsogna, L. Edelstein-Keshet, A.-J. Bernoff, PLoS Comput. Biol. 8, e1002642 (2012).

19. J.G. Puckett, N.T. Ouellette, J. R. Soc. Interface 11, 20140710 (2014).

20. D.J. Thomson, J. Fluid Mech. 180, 529 (1987).

21. Y.-L. Chuang, M.R. D'Orsogna, D. Marthaler, A.L. Bertozzi, L.S. Chayes, Physica D 232, 33 (2007).

22. A.M. Reynolds, D. Reynolds, S.P. Sane, G. Hu, J.W. Chapman, Philos. Trans. R. Soc. B 371, 20150392 (2016).

23. A.M. Reynolds, Phys. Fluids 15, L1 (2003).

24. A.M. Reynolds, Phys. Rev. Lett. 91, 84503 (2003).

25. A.M. Reynolds, N. Mordant, A.M. Crawford, E. Bodenschatz, New J. Phys. 7, 58 (2005).

26. A.G. Lamorgese, S.B. Pope, P.K. Yeung, B.L. Sawford, J. Fluid Mech. 582, 423 (2007).

27. N. Mordant, A.M. Crawford, E. Bodenschatz, Physica D 193, 245 (2004).

28. B.L. Sawford, P.K. Yeung, M.S. Borgas, P. Vedula, La Porta, A.M. Crawford, E. Bodenschatz, Phys. Fluids 15, 3478 (2003).

29. Y.-L. Chuang, T. Chou, M.R. D’Orsogna, Phys. Rev. E 93, 043112 (2016).

30. J.G. Puckett, R. Ni, N.T. Ouellette, Phys. Rev. Lett. 114, 258103 (2015).

31. D. Gorbonos, N.S. Gov, Phys. Rev. E 95, 042405 (2017).

32. N.T. Ouellette, Toward a "thermodynamics" of collective behaviour (SIAM News, 2017).

33. N.M. Smith, A.K. Dickerson, D. Murphy, Bioinspir. Biomim. 14, 031001 (2019). 